\title{
Anderson localisation of Dirac eigenmodes in high temperature QCD
}

\section{Guido Cossu*}

School of Physics and Astronomy, University of Edinburgh, Edinburgh EH9 3FD

Theory Center, IPNS, High Energy Accelerator Research Organization (KEK), Tsukuba, Ibaraki 305-0810, Japan

E-mail: guido.cossu@ed.ac.uk

\section{Shoji Hashimoto}

Theory Center, IPNS, High Energy Accelerator Research Organization (KEK), Tsukuba, Ibaraki 305-0810, Japan

E-mail: shoji.hashimoto@kek.jp

\begin{abstract}
We investigate the properties of background gauge field configurations that act as disorder for the Anderson localization mechanism in the Dirac spectrum of QCD at high temperatures. We compute the eigenmodes of the Möbius domain-wall fermion operator on configurations generated for the $S U(3)$ gauge theory with two flavors of fermions. We identify the source of localization of the eigenmodes with gauge configurations that are approximately self-dual and support local negative fluctuations of the Polyakov loop $P_{L}(x)$, in the bulk of $P_{L} \sim 1$. We investigate the dependence of these observations on the boundary conditions of the valence operator and interpret the results in terms of monopole-instanton structures. The results presented here are from [1], where we describe more details on the methods and conclusions.
\end{abstract}

34th annual International Symposium on Lattice Field Theory

24-30 July 2016

University of Southampton, UK

${ }^{*}$ Speaker. 


\section{Introduction}

The finite temperature phase transition in QCD-like theories separates a regime of confining and spontaneously broken chiral vacuum from the deconfining and chirally symmetric one. In the case of QCD the deconfinement temperature $T_{d}$ and the chiral restoration temperature $T_{c}$ are remarkably close or even conincide with each other, suggesting a deeper connection between two otherwise unrelated properties of the vacuum. Whether there is a mechanism to relate them or not is still an open question.

The eigenvalues and eigenvectors of the QCD Dirac operator provide important means to probe the properties of the vacuum. They control the low energy physics and chiral restoration. In particular their role in the chiral breaking/restoration is well understood. In this proceeding we report our finite-temperature study on the relation between the lowest eigenmodes of the Dirac operator and their supporting background gauge configurations in order to shed some light on the possible connection between confinement and chiral symmetry. A set of important characteristics of the Dirac spectrum are the local fluctuations of the eigenvalues in the bulk and the localisation of the corresponding eigenmodes. The high temperature Dirac spectrum experiences a transition at fixed $T$ between a low-energy region, where the eigenvalues are uncorrelated, and the higher part of the spectrum that exhibits non-trivial Random Matrix Theory (RMT) type of correlations (see $[2,3,4]$ and Section 2 below).

There is a tight connection between the local spectral correlations and the localisation of the modes. It has been proven using staggered and overlap fermions in $[2,3,4,5]$ (and we provide a confirmation using domain-wall fermions in Section 2) that the lowest modes of the high temperature spectrum are localised below the threshold $\lambda_{c}$ where delocalisation occurs.

The authors of [4] have shown that the scaling critical exponents for the QCD spectrum match the expectation from the three dimensional Anderson Hamiltonian model, confirming that there is an Anderson-like mechanism in action in high temperature QCD. The attractive question, once we assume Anderson localisation in, QCD, is what are the impurities, or the disorder, in the background gauge configurations that trigger the localisation of the eigenmodes. How is the diffusion of fermions affected by the impurities? Are these related to the chiral transition and/or deconfinement? These are the central topics of the study.

The interested reader can find a full description of our methodology and results in the published paper [1]. Analogous investigations were reported at this conference [6] and in recent papers [7, 8].

\section{Dirac spectrum and localisation properties}

\subsection{Numerical setup}

We study the properties of the Dirac eigenmodes of two-flavour QCD, using the Möbius domain-wall fermion operator $[9,10]$, on configurations around the phase transition temperature. We simulated several ensembles in the temperature range $[0.9,1.9] T_{d}$ where $T_{d}$ is the deconfinement temperature $\left(T_{d} \simeq T_{c}\right)$, and at several masses. The deconfinement transition temperature is estimated to be $T_{d}=175(5) \mathrm{MeV}$ from the measurement of the Polyakov loop. We have three type of lattice sizes $16^{3} \times 8,32^{3} \times 8$ and $32^{3} \times 12$ to control the dependence on the volume and lattice spacing. More details on the ensembles are in [1]. 


\subsection{Eigenmode localisation}

A basic quantity to understand the localisation properties is the participation ratio (PR) that is defined as

$$
P R_{n}=\left(V \int\left|\psi_{n}(x)\right|^{4} d^{4} x\right)^{-1}, \quad \int\left|\psi_{n}(x)\right|^{2} d^{4} x=1,
$$

where $\psi_{n}$ is the $n$-th lowest eigenmode of the Dirac operator, normalised to $1 . P R_{n}$ is constant $(=1)$ in the purely metallic phase with a completely delocalised eigenfunction, $\left|\psi_{n}(x)\right|^{2}=1 / V$. It is, on the other hand, proportional to $1 / V$ if the mode is completely localised, which implies an insulator phase. With this definition the PR represents the fraction of the total volume occupied by the support of the eigenmode $\psi_{n}$. Therefore the scaling with the volume of the eigenmodes will distinguish the localised and delocalised states.

The scaling of low modes has been already studied in detail by Kovacs et al. using staggered fermions [2, 3]. In [1] we reported a similar analysis using our domain-wall configurations. Here we show the result for the dependence on the boundary conditions of the PR in Figure 1, for one ensemble. The lowest modes for the periodic boundary condition on the same gauge configurations occupy a larger fraction of the volume. It is an indication of a possible delocalisation but this conclusion cannot be inferred without a proper scaling analysis. In the following we will discuss other observables that complete the picture, showing delocalisation of the lowest modes when the boundary conditions are changed.

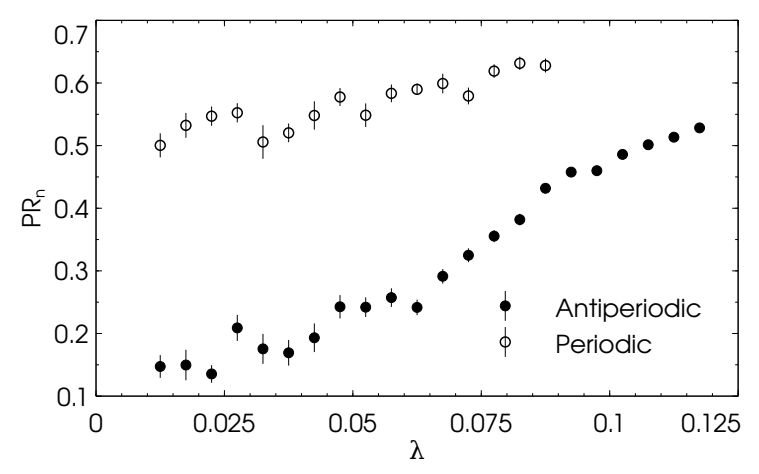

Figure 1: Dependence of the participation ratio on the boundary conditions for the ensemble $32^{3} \times 12$, $\beta=4.30, m=0.01$. The corresponding temperature is $T=220 \mathrm{MeV}$ and the bare quark mass is $26 \mathrm{MeV}$.

\subsection{Level spacing distribution}

The unfolded level spacing distribution (ULSD) is another trait that distinguishes the insulator and metallic phases in the Anderson model, together with localisation. The ULSD is the probability distribution $P(s)$ of

$$
s_{i}=\frac{\lambda_{i+1}-\lambda_{i}}{\left\langle\lambda_{i+1}-\lambda_{i}\right\rangle}
$$

the distance of two consecutive eigenvalues in the unfolded spectrum of a matrix [11]. Completely decorrelated eigenvalues are expected to be distributed according to the Poisson distribution. The 
insulator phase of the Anderson model, with strong disorder and localisation, has Poisson distributed eigenvalues while the weak disorder and metallic phase show delocalised modes distributed according to RMT. In this case the ULSD can be analytically computed.

By universality arguments the low temperature chirally broken phase in QCD is expected to follow the predictions of one of the RMT ensembles, the GUE in our case. This has been extensively tested, for examples see the review [12] and our data in [1]. By changing the boundary condition on the same ensemble, right panel of Figure 2, the ULSD changes radically and it is in accordance with RMT, similar to the low temperature case. This is expected from our discussion on the PR. In our perspective it poses the question of how the eigenmode localisation depends on the boundary conditions. The background gauge field is unchanged. The lattice physical dimensions are unchanged. But the modes are now delocalised. In the following we investigate this difference.
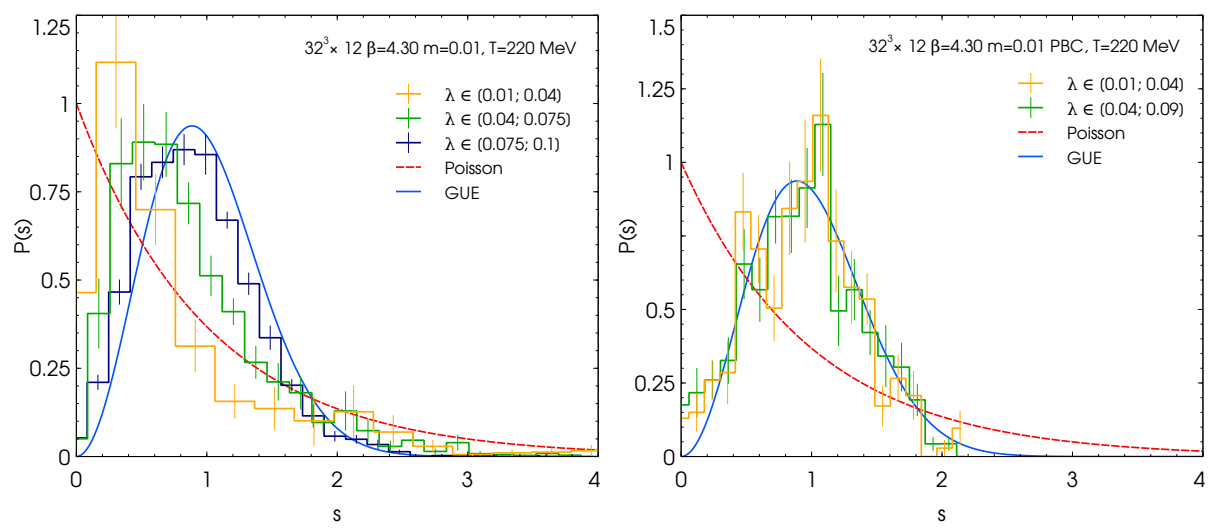

Figure 2: Distribution $P(s)$ of the level spacings for the unfolded Dirac spectrum in several sectors. The left panel is for the high temperature $T=220 \mathrm{MeV}$ case. The right panel shows the result on the same configurations but with the valence Dirac operator having periodic boundary conditions. Continuous lines are the analytic predictions for totally uncorrelated eigenvalues (Poisson, red-dashed curve) and random matrix theory from the unitary ensemble (GUE, blue curve), with no free parameters.

\section{Numerical analysis of the background gauge field}

We now discuss the numerical results on the background gauge field supporting the eigenmodes, with the intent of identifying the source of localisation. We begin with the investigation of the correlation of the eigenmodes with the trace of the Polyakov line, or holonomy, in a gauge theory with $N$ colors $P_{L}(x)=\frac{1}{N} \operatorname{Tr} \exp \left(i \oint A_{4} \mathrm{~d} \tau\right), \tau$ being the compact temporal dimension. This is a gauge invariant quantity related to confinement. Similar studies on this observable were conducted by other groups on pure gauge configurations or using effective models [5, 13]. The Polyakov line is measured after suppressing UV fluctuations with the Wilson flow. We then correlate, for several temperatures and masses, the local norm of the non-zero eigenmodes $|\psi(x)|^{2}$, with the real part of the Polyakov line trace. An example of the results is reported in Figure 3. The lowest eigenmodes are localised, i.e. the norm is higher, where the Polyakov line is mostly negative. The highest modes, delocalised, show no particular dependence on $\operatorname{Re} P_{L}(x)$. The situation changes when we change the boundary conditions, right panel of Figure 3 of the valence Dirac operator. The low modes are not tied anymore to any particular value of the Polyakov loop, as the higher modes. 

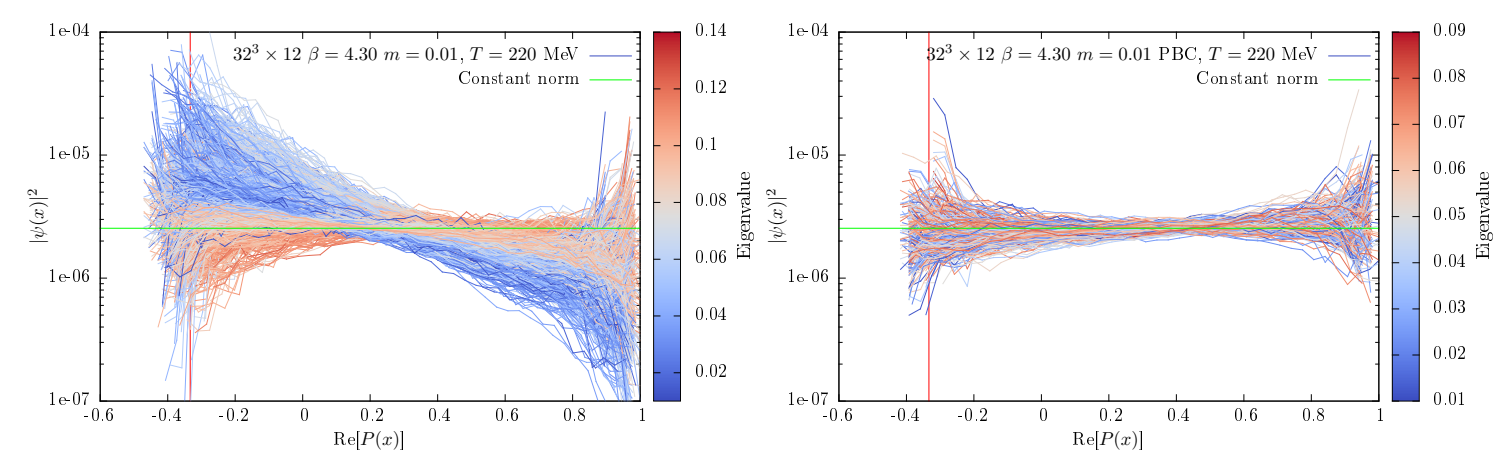

Figure 3: Two examples of the local squared norm of the eigenmode in correlation with the local Polyakov line (real part). The colours represent the eigenvalue, as indicated by the colour bar attached to each plot. The green horizontal line represents a vector with a constant norm. Log scale for the norm axis is used. The vertical red line indicates the location of $\operatorname{Re} P_{L}=-1 / 3$. Right panel: valence operator with periodic boundary conditions.

Two other relevant gauge invariant quantities that characterise the background configuration are the action density $s(x)=F_{\mu v} F_{\mu \nu}(x)$ and the topological charge density $q(x)=F_{\mu v} \tilde{F}_{\mu v}(x)$, without the canonical constant $8 / 32 \pi^{2}$ in front. For a self-dual field $\tilde{F}=F$, the ratio $|q| / s=1$. Figure 5 shows that the lower modes are related to regions where the action is higher than the average and the configurations are self dual. See the full paper for a more detailed discussion [1].
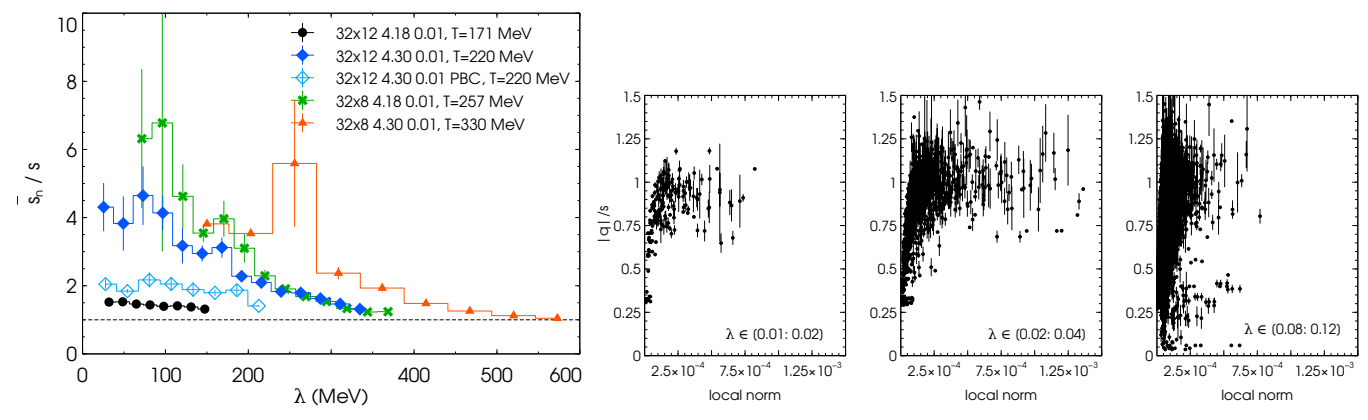

Figure 4: Ratio of the eigenmode weighted average of the action $\bar{s}_{n}$ and the average $s$, in correlation with the eigenvalue. Lowest modes favour higher action regions. Temperatures ranging from $[0.9,1.9] T_{c}$.

Figure 5: Dependence of the $|q| / s$ ratio on the norm for several eigenvalue sectors. Ensemble $32^{3} \times 12$, $\beta=4.30, m=0.01, T=220 \mathrm{MeV}$.

The picture that arises from the analysis of the correlations of the low modes with these quantities is that lowest modes are localised where the Polyakov loop is negative and precisely in the region where only two of its eigenvalues are equal and negative $\sim-1$, as discussed above. The same regions are characterised by an action several times higher than the average and an almost self dual gauge field. These are some gauge invariant properties of the underlying fluctuations supporting the low modes at finite temperature. On the same configurations the lowest modes of the operator with periodic boundary condition show a much milder dependence, if none, on these features. The localisation mechanism depends on the boundary conditions. 


\begin{tabular}{|c|c|c|c|c|}
\hline & $M_{m}$ & $\bar{M}_{m}$ & $L$ & $\bar{L}$ \\
\hline E-charge & + & + & $-(N-1)$ & $-(N-1)$ \\
B-charge & + & - & $-(N-1)$ & $+(N-1)$ \\
action S, $\frac{8 \pi^{2}}{g^{2}}$ & $v_{m}$ & $v_{m}$ & $1-\sum v_{m}$ & $1-\sum v_{m}$ \\
top. charge Q & $v_{m}$ & $-v_{m}$ & $1-\sum v_{m}$ & $\sum v_{m}-1$ \\
\hline
\end{tabular}

Table 1: List of characteristics of the self-dual solutions for $S U(N)$ groups. Here the index $m \in[1 \ldots N-1]$, distinguishes the BPS ( $M$-type) monopoles. $v_{m}$ phase is in unit of $2 \pi$ is the difference of two consecutive eigenvalues of $P_{L}$. The bar denotes the anti-monopoles solutions. $L$-type monopoles are Kaluza-Klein monopoles. A $Q=1$ caloron is composed by the sum of the different $N-1 M$-type monopoles and the $L$-type. It is neutral and has the canonical action $\frac{8 \pi^{2}}{g^{2}} . L$ monopoles have B-charge \pm 1 in each one of the $N-1$ Cartan subgroups.

\section{Interpretation in terms of topological objects}

We now turn to an interpretation of the results presented in the previous sections. Anticipating in short our conclusions, the background configurations supporting the near-zero modes have the characteristics of monopole-instanton (sometime called dyon in the literature) pairs. The monopole instantons are self-dual solutions of the Yang-Mills equations of motion and the constituents of the calorons, the extension of the instanton solution to a background with non-trivial Polyakov line. In [1] we discuss the properties of the monopole-instantons (see also [14] for a review). A summary is presented in table 1. In the following we will use the terminology defined in this table.

The relation between the calorons and the zero modes of the Dirac operator has been explored analytically in $[15,16,17,18]$. A remarkable result of these papers is the dependence of the eigenmode location on the boundary conditions of the fermionic field. The general result states that for the boundary condition $\psi(t+T)=\exp (2 \pi i \phi) \psi(t)$ the eigenmode is localised on the monopole $m$ that satisfies $\phi \in\left[\mu_{m}, \mu_{m+1}\right], \mu_{m}$ is the m-th eigenvalue of $P_{L}(\infty)$, see [1]. At high temperature periodic zero modes are localised on the light $M$-constituents while anti-periodic zero-modes localise around the heavier $L$ monopoles. The results of this paper suggest that these properties of the zero-modes for the classical Yang-Mills (YM) background do apply also for low-lying non-zero modes. The underlying assumption is that topological fluctuations are stable under deformations.

The numerical evidences in Section 3, on the properties of the background configurations supporting the low-lying modes with anti-periodic boundary conditions, indicate that these modes are localised around the heavy KK monopole fluctuations of the gauge field.

In conclusion, we showed using chiral fermions that, mode by mode, the disorder underlying the Anderson localisation is composed by $L$-type monopoles which have increasing action with the temperature, and this is reflected by the increasing localisation of the near-zero modes. At finite temperature these heavy $L$-type monopoles excitations are expected to be suppressed while most of the lattice points support lighter fluctuations.

We presented several numerical evidences that the background gauge configurations supporting each near-zero eigenmode of the Dirac operator in the fundamental representation are self-dual and carry higher action than the volume average. They are strongly related to fluctuations of the Polyakov line where two of the eigenvalues of the holonomy are identical (equal to -1 at high 
temperature). The data show localisation of the lowest eigenmodes in such regions of negative Polyakov line, large action and self-dual gauge fields. Changing the boundary conditions of the Dirac operator on the same finite temperature ensembles washes up the localised states and all modes become delocalised.

\section{References}

[1] G. Cossu and S. Hashimoto, Anderson Localization in high temperature QCD: background configuration properties and Dirac eigenmodes, JHEP 06 (2016) 056, [1604 . 00768].

[2] T. G. Kovács and F. Pittler, Anderson localization in quark-gluon plasma, Phys. Rev. Lett. 105 (Nov, 2010) 192001.

[3] T. G. Kovács and F. Pittler, Poisson-random matrix transition in the qcd dirac spectrum, Phys. Rev. D 86 (Dec, 2012) 114515.

[4] M. Giordano, T. G. Kovacs and F. Pittler, Universality and the QCD Anderson Transition, Phys. Rev. Lett. 112 (2014) 102002, [1312.1179].

[5] F. Bruckmann, T. G. Kovacs and S. Schierenberg, Anderson localization through Polyakov loops: lattice evidence and Random matrix model, Phys. Rev. D84 (2011) 034505, [1105. 5336].

[6] M. Giordano, T. G. Kovacs and F. Pittler, Chiral transition, eigenmode localisation and Anderson-like models, in Proceedings, 34th International Symposium on Lattice Field Theory (Lattice 2016): Southampton, UK, July 24-30, 2016, 2016. 1610.07412.

[7] M. Giordano, T. G. Kovacs and F. Pittler, An Anderson-like model of the QCD chiral transition, 1603.09548.

[8] M. Giordano, S. D. Katz, T. G. Kovacs and F. Pittler, Deconfinement, chiral transition and localisation in a QCD-like model, 1611.03284.

[9] R. Brower, H. Neff and K. Orginos, Mobius fermions, Nucl.Phys.Proc.Suppl. 153 (2006) 191-198, [hep-lat/0511031].

[10] R. C. Brower, H. Neff and K. Orginos, The Móbius Domain Wall Fermion Algorithm, 1206.5214.

[11] G. Akemann, J. Baik and P. Di Francesco, eds., The Oxford Handbook of Random Matrix Theory. Oxford University Press, 2011.

[12] J. J. M. Verbaarschot and T. Wettig, Random matrix theory and chiral symmetry in QCD, Ann. Rev. Nucl. Part. Sci. 50 (2000) 343-410, [hep-ph/ 0003017 ].

[13] M. Giordano, T. G. Kovacs and F. Pittler, An Ising-Anderson model of localisation in high-temperature QCD, JHEP 04 (2015) 112, [1502 . 02532].

[14] D. Diakonov, Topology and confinement, Nucl. Phys. Proc. Suppl. 195 (2009) 5-45, [0 906.2456 ].

[15] M. Garcia Perez, A. Gonzalez-Arroyo, C. Pena and P. van Baal, Weyl-Dirac zero mode for calorons, Phys. Rev. D60 (1999) 031901, [hep-th/9905016].

[16] M. N. Chernodub, T. C. Kraan and P. van Baal, Exact fermion zero mode for the new calorons, Nucl. Phys. Proc. Suppl. 83 (2000) 556-558, [hep-lat/9907001].

[17] T. M. W. Nye and M. A. Singer, An $L^{* * 2}$ index theorem for Dirac operators on $S^{* *} 1 \times R^{* * 3}$, Submitted to: J. Funct. Anal. (2000), [math/0009144].

[18] E. Poppitz and M. Unsal, Index theorem for topological excitations on $R^{* * 3} \times S^{* *} I$ and Chern-Simons theory, JHEP 03 (2009) 027, [0 812 . 2085]. 